\title{
Transoral Laser Surgery (Glottis)
}

National Cancer Institute

\section{Source}

National Cancer Institute. Transoral Laser Surgery (Glottis). NCI Thesaurus. Code C91851.

An endoscopic laser microsurgical laryngeal procedure for patients with early glottis tumors. 